PROCEEDINGS OF THE

AMERICAN MATHEMATICAL SOCIETY

Volume 135, Number 10, October 2007, Pages 3073-3082

S 0002-9939(07)08879-X

Article electronically published on May 14, 2007

\title{
ON THE DEGREE OF HILBERT POLYNOMIALS ASSOCIATED TO THE TORSION FUNCTOR
}

\author{
DANIEL KATZ AND EMANOIL THEODORESCU
}

(Communicated by Bernd Ulrich)

\begin{abstract}
Let $R$ be a local, Noetherian ring and $I \subseteq R$ an ideal. A question of Kodiyalam asks whether for fixed $i>0$, the polynomial giving the $i$ th Betti number of $I^{n}$ has degree equal to the analytic spread of $I$ minus one. Under mild conditions on $R$, we show that the answer is positive in a number of cases, including when $I$ is divisible by $\mathfrak{m}$ or $I$ is an integrally closed $\mathfrak{m}$-primary ideal.
\end{abstract}

\section{INTRODUCTION}

Let $(R, \mathfrak{m}, k)$ be a local ring with maximal ideal $\mathfrak{m}$ and residue field $k$. In this note we wish to study the degree of Hilbert-Samuel polynomials of the form $\tau_{i}(n):=$ $\lambda\left(\operatorname{Tor}_{i}^{R}\left(R / I^{n}, M\right)\right)$, where $I \subseteq R$ is an ideal, $M$ is a finitely generated $R$-module and $\lambda(T)$ denotes the length of the $R$-module $T$. These polynomials represent generalizations of the standard Hilbert-Samuel polynomial $P(n):=\lambda\left(C / I^{n} C\right)$, where $C$ is a finitely generated module for which $\lambda(C / I C)$ is finite. In this case, it is well known that the degree of $P(n)$ equals $\operatorname{dim}(C)$ and its normalized leading coefficient is the multiplicity of $I$ on $C$. However, for the polynomial $\tau_{i}(n)$, the current state of affairs is not very well understood, though there are some partial results. It is not hard to see that $\tau_{i}(n)$ has degree at most $\ell(I)-1$, where $\ell(I)$ denotes the analytic spread of $I$ (see Remark 3.2 below). Kodiyalam asks whether in the case $M=k$ the degree of $\tau_{i}(n)$ might equal $\ell(I)-1$, provided the Tor modules in question do not vanish. For values of $M$ different from $k$, one has mixed expectations regarding the degree of $\tau_{i}(n)$. For example, in [3, Theorem I], it is shown that if $R$ is Cohen-Macaulay and $I=\mathfrak{m}$, then the $\tau_{i}(n)$ has the expected degree $d-1$; but an example ([3], Proposition III) is given showing that if $R$ is not Cohen-Macaulay, then the degree can be less than $d-1$. As we shall see below, the relevant point regarding the degree of $\tau_{i}(n)$ seems to be that the syzygies of $M$ have maximal dimension, so we are able to recover the result from 3 assuming only that $R$ is unmixed and equidimensional on the one hand and offer an explanation of their example on the other. More precisely, our main results, Theorem 3.3 and Theorem 3.4, show that as long as syzygies over $R$ are reasonably well behaved, then the degree of $\tau_{i}(n)$ is $\ell(I)-1$ when we replace the filtration $\left\{I^{n}\right\}$ by an $I$ good filtration $\left\{J_{n}\right\}$ which either satisfies $J_{n}$ is divisible by $\mathfrak{m},\left(\mathfrak{m} J_{n}: \mathfrak{m}\right)=J_{n}$ or

Received by the editors December 14, 2005, and, in revised form, June 28, 2006.

2000 Mathematics Subject Classification. Primary 13A30, 13D02, 13D07, 13 D40.

Key words and phrases. Hilbert-Samuel polynomial, torsion functor, quasi-unmixed local ring.

(C)2007 American Mathematical Society

Reverts to public domain 28 years from publication 3073 
$\left(J_{n}: \mathfrak{m}\right) \nsubseteq \overline{J_{n}}$, where the overbar denotes integral closure. The results stated in the abstract are then immediate corollaries.

\section{Preliminaries}

In this section we set our notation and lay the groundwork for our main results concerning the degree of the Hilbert-Samuel polynomials under consideration. Our primary motivation and interest is the original question of Kodiyalam concerning the asymptotic behavior of the $i$ th Betti numbers of powers of a fixed ideal, i.e., the case when $M=k$. On the other hand, it is not yet clear to us whether there is anything particularly special about $k$, except that its syzygies have nilpotent annihilators.

Throughout $(R, \mathfrak{m}, k)$ will denote a Noetherian local ring having residue field $k$ and Krull dimension $d$. Given an ideal $I \subseteq R$ and a finitely generated $R$-module $M$, we assume that $I$ is not nilpotent and we let $\tau_{i}^{I}(n)$ or just $\tau_{i}(n)$ denote the polynomial giving the lengths of $\operatorname{Tor}_{i}^{R}\left(R / I^{n}, M\right)$ for $n$ large, when such lengths are finite. We shall tacitly assume that $i \leq$ p. d. $(M)$. Similarly, if $\mathcal{J}:=\left\{J_{n}\right\}_{n \geq 0}$ is a filtration such that the lengths of the modules $\operatorname{Tor}_{i}^{R}\left(R / J_{n}, M\right)$ take on the values of a polynomial for $n$ large, we write $\tau_{i}^{\mathcal{J}}(n)$ or just $\tau_{i}(n)$ for this polynomial. The motivating case for us is the case when $M=k$, in which case $\tau_{i}(n)$ gives the $i$ th Betti numbers of $R / I^{n}$ for $n$ large. By dimension shifting, if $\operatorname{deg}\left(\tau_{i}(n)\right)=\ell(I)-1$, then the polynomial giving the degree of the $i$ th Betti numbers of $I^{n}$ also has degree $\ell(I)-1$.

For an ideal $I$, we will consider filtrations of ideals $\mathcal{J}:=\left\{J_{n}\right\}_{n \geq 0}$ satisfying the following properties : (i) $I^{n} \subseteq J_{n}$, for all $n$, (ii) $I^{n} \cdot J_{m} \subseteq J_{n+m}$, for all $n$ and $m$, and (iii) there exists $q \geq 1$ such that $J_{n+q}=I^{n} J_{q}$ for all $n$. We will say that such a filtration is a good filtration with respect to $I$. Note that we are not requiring that $J_{n} \cdot J_{m} \subseteq J_{n+m}$. We write $\mathcal{R}_{I}:=\bigoplus_{n \geq 0} I^{n} t^{n}$ for the Rees ring of $R$ with respect to $I$ ( $t$ an indeterminate) and, by abuse of notation, $\mathcal{R}_{\mathcal{J}}:=\bigoplus_{n \geq 0} J_{n} t^{n}$ for the finite $\mathcal{R}_{I}$-module corresponding to the filtration $\mathcal{J}$.

We will use the following notation. If $I \subseteq R$ is an ideal and $K$ is a finitely generated $R$-module, then we write $\ell_{K}(I)$ to denote the analytic spread of $I$ on $K$, which is just the Krull dimension of the graded module $\bigoplus_{n \geq 0} I^{n} K / \mathfrak{m} I^{n} K$. When $K=R$, we write $\ell(I)$.

We begin with a remark concerning syzygies over $R$. The contents are well known to experts, but we include them for the convenience of the reader and for ease of reference.

Remark 2.1. (i) Suppose that $M$ has a rank; in other words, there exists an $r \geq 0$ such that $M_{Q}$ is a free $R_{Q}$-module of rank $r$ for all $Q \in \operatorname{Ass}(R)$. Then any non-zero syzygy $K$ of $M$ is a faithful $R$-module. To see this, note that $K_{Q}$ is a free $R_{Q}$ module of constant rank, for all $Q \in \operatorname{Ass}(R)$. Thus, for any such $Q, K_{Q}=0$ if and only if $K=0$. For any $x \in R$, if $x \cdot K=0$, then $x \cdot K_{Q}=0$; thus, $x_{Q}=0$. Since this holds for all $Q, x=0$, so $K$ is faithful. In particular, it follows that if $\operatorname{depth}(R)>0$, then every syzygy of every finite length $R$-module, and hence of $k$, is faithful.

(ii) Suppose $\operatorname{dim}(R)>0$ and $\operatorname{depth}(R)=0$. Let $K$ be the $i$ th syzygy in a minimal free resolution of the residue field of $R$. We note that the annihilator of $K$ is nilpotent. Indeed, for all primes $Q \neq \mathfrak{m}, K_{Q}$ is free of some fixed rank. 
If this rank were zero, then $K$ would have finite length. By [5, Corollary 3.16], $\operatorname{Tor}_{i}^{R}\left(R / \mathfrak{m}^{n+1}, k\right)=\mathfrak{m}^{n} K / \mathfrak{m}^{n+1} K$, for $n$ large, so this Tor vanishes for large $n$, which is absurd, for then p.d. $\left(R / \mathfrak{m}^{n+1}\right)$ would be finite. Thus $K_{Q} \neq 0$ for all primes $Q \neq \mathfrak{m}$. It follows that the annihilator of $K$ is nilpotent. Note that the annihilator of $K$ is a non-zero nilpotent ideal, since the same clearly holds for $\mathfrak{m}$. In Theorem 3.3 below, we will show that the syzygies of any integrally closed $\mathfrak{m}$-primary ideal have nilpotent annihilators.

As mentioned above, a key ingredient in our main results is the dimension of the syzygies over $R$. More precisely, this ingredient will be the dimension of various graded modules over $\mathcal{R}_{I}$ determined by the syzygy and the filtration under consideration. The propositions below address this issue.

Let $I \subseteq R$ be an ideal and $K$ a finitely generated $R$-module. Then it is well known that $\operatorname{dim}\left(\bigoplus_{n \geq 0} I^{n} K / I^{n+1} K\right)=\operatorname{dim}(K)$ and $\ell_{K}(I)=\ell_{R / a n n(K)}(I)$. Our first proposition notes that these relations extend to filtrations that are good with respect to $I$. This result follows immediately from the ideal case, but lacking a suitable reference, we give a brief indication of the proof.

Proposition 2.2. Let $I \subseteq R$ be an ideal and $\mathcal{J}:=\left\{J_{n}\right\}$ be an $I$-good filtration. Let $K$ be a finitely generated $R$-module. Then : (i) $\operatorname{dim}\left(\bigoplus_{n \geq 0} J_{n} K / J_{n+1} K\right)=\operatorname{dim}(K)$ and (ii) $\operatorname{dim}\left(\bigoplus_{n \geq 0} J_{n} K / \mathfrak{m} J_{n} K\right)=\ell_{K}(I)$.

Proof. We may mod out the annihilator of $K$ and assume that $K$ is a faithful $R$ module. Set $\mathcal{S}:=\mathcal{R}_{I}\left[t^{-1}\right]$ and $\mathcal{K}:=\bigoplus_{-\infty}^{\infty} J_{n} K t^{n}\left(J_{n}:=R\right.$ if $\left.n \leq 0\right)$, so that $\mathcal{K}$ is a finitely generated, faithful $\mathcal{S}$-module and $\mathcal{K}_{\geq 0}$ is a finitely generated, faithful

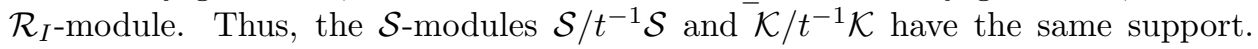
Similarly, the $\mathcal{R}_{I}$-modules $\mathcal{R}_{I} / \mathfrak{m} \mathcal{R}_{I}$ and $\mathcal{K}_{\geq 0} / \mathfrak{m} \mathcal{K}_{\geq 0}$ have the same support. The required identities now follow from the definitions and the well-known fact that $\operatorname{dim}\left(\mathcal{S} / t^{-1} \mathcal{S}\right)=\operatorname{dim}(R)$.

In the proof of the following proposition we will use the fact that if $R$ is a quasiunmixed local ring, then $\mathcal{R}_{\mathcal{J}}\left[t^{-1}\right]$ localized at its homogeneous maximal ideal is a quasi-unmixed local ring of dimension $d+1$ (see [8], Proposition 2.1).

Proposition 2.3. Let $K$ be a finitely generated $R$-module with nilpotent annihilator. Let $J_{n}$ be an I-good filtration and assume $\mathcal{R}_{\mathcal{J}}$ is a ring. Let $\mathcal{L}:=\left\{L_{n}\right\}$ be another filtration satisfying: (i) $J_{n} \subseteq L_{n}$, for all $n$, (ii) $\mathcal{R}_{\mathcal{L}}$ is an $\mathcal{R}_{\mathcal{J}}$-module, (iii) $x \cdot \mathcal{R}_{\mathcal{L}} \subseteq \mathcal{R}_{\mathcal{J}}$, for some non-zerodivisor $x \in R$ and (iv) $L_{q} \not \subseteq \overline{I^{q}}$, for some $q$. Assume furthermore that $R$ is quasi-unmixed. Then $\operatorname{dim}\left(\bigoplus_{n \geq 0} L_{n} K / J_{n} K\right)=d$, where $d=\operatorname{dim}(R)$.

Proof. Set $\mathcal{S}:=\mathcal{R}_{\mathcal{J}}\left[t^{-1}\right]=\bigoplus_{-\infty}^{\infty} J_{n} t^{n}$ and write $\mathcal{M}$ for its homogeneous maximal ideal. Since $\mathcal{C}:=\bigoplus_{n \geq 0} L_{n} K / J_{n} K$ is the quotient of the finite $\mathcal{S}$-module $\bigoplus_{-\infty}^{\infty} L_{n} K t^{n}$ by its submodule $\bigoplus_{-\infty}^{\infty} J_{n} K t^{n}, \mathcal{C}$ is a finite module $\mathcal{S}$-module, and thus its dimension is the same over both rings $\mathcal{R}_{\mathcal{J}}$ and $\mathcal{S}$. We are going to regard $\mathcal{C}$ as a module over the $\operatorname{ring} \mathcal{S}$. Let $\mathcal{A} \subseteq \mathcal{S}$ denote the annihilator of $\mathcal{C}$. Since $\mathcal{A}$ contains the non-zerodivisor $x$, we have $\operatorname{dim}(\mathcal{C})=\operatorname{dim} /(\mathcal{S} / \mathcal{A}) \leq d$.

Now let $f t^{r} \in \mathcal{A}$ be a homogeneous element. (Note, $r \in \mathbb{Z}$.) Then for all $n$, we have $f L_{n} K \subseteq J_{n+r} K$. If $a \in f L_{n}$, then the usual determinant trick shows that $a$ is integral over the ideal $J_{n+r}$ modulo the annihilator of $K$. But then $a$ is integral over $J_{n+r}$, since the annihilator of $K$ is nilpotent. Thus, $f L_{n} \subseteq \overline{J_{n+r}}$. It 
follows that $\mathcal{A} \cdot \mathcal{R}_{\mathcal{L}}\left[t^{-1}\right] \subseteq \overline{\mathcal{S}}$. By hypothesis, we can write $\mathcal{R}_{\mathcal{L}}\left[t^{-1}\right]=\frac{\mathcal{U}}{x}$, for some ideal $\mathcal{U} \subseteq \mathcal{S}$. Thus, $\mathcal{A} \cdot \mathcal{U} \subseteq x \cdot \overline{\mathcal{S}}$, from which it follows that $\mathcal{A} \cdot \mathcal{U} \subseteq \overline{x \cdot \mathcal{S}}$. If $\mathcal{U} \subseteq \overline{x \cdot \mathcal{S}}$, then $\frac{\mathcal{U}}{x} \subseteq \overline{\mathcal{S}}$. But this would imply $L_{q} \subseteq \overline{J_{q}}=\overline{I^{q}}$, a contradiction. Thus, $\mathcal{A}$ consists of zerodivisors modulo the ideal $\overline{x \cdot \mathcal{S}}$, so $\mathcal{A} \subseteq \mathcal{P}$, for some prime $\mathcal{P}$ in $\mathcal{S}$ associated to $\overline{x \cdot \mathcal{S}}$. Since $\overline{x \cdot \mathcal{S}}$ is homogeneous, $\mathcal{P}$ is contained in $\mathcal{M}$. Since $R$ is quasi-unmixed, $\mathcal{S}_{\mathcal{M}}$ is quasi-unmixed, so $\operatorname{height}(\mathcal{P})=1$ ([9], Theorem 2.12). It follows that $\operatorname{dim}(\mathcal{S} / \mathcal{P})=d$, so $\operatorname{dim}(\mathcal{S} / \mathcal{A})=d$, as required.

\section{THE DEGREE OF $\tau_{i}(n)$}

In this section we present our main results concerning the degree of $\tau_{i}^{\mathcal{J}}(n)$. We provide three classes of filtrations of ideals for which $\tau_{i}^{\mathcal{J}}(n)$ (with $M$ essentially arbitrary) has maximal degree. In particular, our main results apply to filtrations satisfying any one of the following conditions : $J_{n}$ is divisible by $\mathfrak{m},\left(\mathfrak{m} J_{n}: \mathfrak{m}\right)=J_{n}$ or $\left(J_{n}: \mathfrak{m}\right) \nsubseteq \overline{J_{n}}$. These results in turn provide a large number of examples where the question of Kodiyalam has a positive answer.

Before turning to our main result, we offer a couple of comments and results concerning the degree of $\tau_{i}^{I}(n)$, when $M=k$. In [4], it is shown that for a finite $R$-module $N$ satisfying $\operatorname{dim}(N)=\operatorname{dim}(R)$, the Betti numbers of $\mathfrak{m}^{n} N$ are given by a polynomial of degree $\ell_{N}(\mathfrak{m})-1$, for $n$ large. In particular, $\operatorname{deg}\left(\tau_{i}^{\mathfrak{m}}(n)\right)=d-1$. Kodiyalam also shows that if $I$ is the ideal of maximal minors of a generic $t \times(t+1)$ matrix, the Betti numbers of $I^{n}$ are given by a polynomial of degree $\ell(I)-1$ and cites a result due to Herzog which states that if $R$ is a regular local ring and $\mathcal{R}_{I}$ is Cohen-Macaulay, then the Betti numbers of $I^{n}$ are given by a polynomial of degree $\ell(I)-1$ for $n$ large. These are the only results concerning the case $M=k$ that we know of. In fact, aside from the result of Marley mentioned in the proof of Proposition 3.1 below and Theorem I from [3], these represent the only prior results we know of giving the exact degree of $\tau_{i}^{I}(n)$ for any sort of module $M$.

Proposition 3.1. Let $I \subseteq R$ be an ideal and assume $M=k$. Then $\tau_{i}^{I}(n)$ has maximal degree $\ell(I)-1$ in each of the following cases:

(1) $i=1$;

(2) $R$ is Cohen-Macaulay, $I \subseteq R$ is $\mathfrak{m}$-primary and $1 \leq i \leq d$;

(3) $i=2$, grade $(I) \geq \ell(I)-1$ and $I$ is not generated by a regular element.

Proof. For (1), we note that the short exact sequence $0 \rightarrow \mathfrak{m} \rightarrow R \rightarrow k \rightarrow 0$ gives rise to the exact sequence

$$
0 \rightarrow \operatorname{Tor}_{1}^{R}\left(R / I^{n}, k\right) \rightarrow \mathfrak{m} / \mathfrak{m} I^{n} \rightarrow R / I^{n} \rightarrow k \rightarrow 0,
$$

from which it follows that $\operatorname{Tor}_{1}^{R}\left(R / I^{n}, k\right)=\left(I^{n} \cap \mathfrak{m}\right) / \mathfrak{m} I^{n}=I^{n} / \mathfrak{m} I^{n}$. Thus, we have $\operatorname{deg}\left(\tau_{1}^{I}(n)\right)=\ell(I)-1$, essentially by the definition of analytic spread. For (2), we may harmlessly assume that $k$ is infinite. Let $J$ be a minimal reduction of $I$. Then it is shown in [6, Corollary 4.9], that the degree of the polynomial giving the lengths of $\operatorname{Tor}_{i}^{R}\left(R / I^{n}, R / J\right)$ equals $d-1$, for $1 \leq i \leq d$. Take a short exact sequence of the form $0 \rightarrow k \rightarrow R / J \rightarrow C \rightarrow 0$, where $C$ is a finite length $R$-module. Fix $1 \leq i \leq d$. Using subadditivity of lengths in the associated long exact Tor sequence, it follows that either the degree of the polynomial giving the lengths of $\operatorname{Tor}_{i}^{R}\left(R / I^{n}, k\right)$ equals $d-1$ or the degree of the polynomial giving the lengths of $\operatorname{Tor}_{i}^{R}\left(R / I^{n}, C\right)$ equals $d-1$. If it's the former, we're done. If it's the latter, we repeat the process until $C=k$, and this gives what we want. 
For (3), first note that $\operatorname{Tor}_{2}^{R}\left(R / I^{n}, k\right)=0$ for all large $n$ if and only if $R / I^{n}$ has projective dimension one if and only if $I^{n}$, and hence $I$, is generated by a nonzerodivisor. So if $I$ is not generated by a regular element, we may assume that the Tor modules in question do not vanish.

Now $\operatorname{Tor}_{2}\left(R / I^{n}, k\right)=\operatorname{Tor}_{1}\left(R / I^{n}, \mathfrak{m}\right)$. Assuming this doesn't vanish, we need to prove $\operatorname{deg}\left(\tau_{2}(n)\right)=\ell(I)-1$. We induct on $\ell:=\ell(I)$. If $\ell=1$, then $\operatorname{deg}\left(\tau_{i}(n)\right) \leq 0$, and since $\tau_{2}(n)$ is not identically zero, the result is immediate.

Assume $\ell \geq 2$. Consider the part of the minimal resolution of $\mathfrak{m}$ :

$$
0 \rightarrow K \rightarrow F \rightarrow \mathfrak{m} \rightarrow 0
$$

Pick $x \in I$ a non-zerodivisor so that $x$ is a superficial element of $I$ on $\mathfrak{m}$ and on its first syzygy $K$. This is possible because grade $(I) \geq \ell(I)-1>0$. Let $S$ denote the $\operatorname{ring} R / x R$ and $\mathfrak{n}$ its maximal ideal. We can further choose $x$ so that its image in the fiber ring of $I$ is part of a transcendence basis over $k$. We then have $\ell(I S)=\ell(I)-1$, so grade $(I S) \geq \ell(I S)-1$. The short exact sequence induced by multiplication by $x$,

$$
0 \rightarrow R / I^{n} \stackrel{\cdot x}{\rightarrow} R / I^{n+1} \rightarrow S / I^{n+1} S \rightarrow 0
$$

gives rise to the long exact sequence

$$
\cdots \stackrel{\cdot x}{\rightarrow} \operatorname{Tor}_{1}^{R}\left(R / I^{n+1}, \mathfrak{m}\right) \rightarrow \operatorname{Tor}_{1}^{R}\left(S / I^{n+1} S, \mathfrak{m}\right) \rightarrow \mathfrak{m} / I^{n} \mathfrak{m} \stackrel{\cdot x}{\rightarrow} \mathfrak{m} / I^{n+1} \mathfrak{m} .
$$

As in the proof of [3. Lemma 2.5], the maps in this sequence induced by multiplication by $x$ are injective. It follows that

$$
\lambda\left(\operatorname{Tor}_{1}^{R}\left(R / I^{n+1}, \mathfrak{m}\right)\right)-\lambda\left(\operatorname{Tor}_{1}^{R}\left(R / I^{n}, \mathfrak{m}\right)\right)=\lambda\left(\operatorname{Tor}_{1}^{R}\left(S / I^{n+1} S, \mathfrak{m}\right)\right) .
$$

It now suffices to check that $\operatorname{deg} \lambda\left(\operatorname{Tor}_{1}^{R}\left(S / I^{n+1} S, \mathfrak{m}\right)\right)=\ell(I S)-1$. Our grade hypothesis and choice of $x$ allow us to apply the change of rings formula for Tor, giving

$$
\operatorname{Tor}_{1}^{R}\left(S / I^{n+1} S, \mathfrak{m}\right)=\operatorname{Tor}_{1}^{S}\left(S / I^{n+1} S, \mathfrak{m} / x \mathfrak{m}\right)
$$

Thus, we must prove that the degree of the polynomial giving the lengths of this second Tor module equals $\ell(I S)-1$, the maximum possible value. Consider the short exact sequence of $S$-modules,

$$
0 \rightarrow k \rightarrow \frac{\mathfrak{m}}{x \mathfrak{m}} \rightarrow \frac{\mathfrak{m}}{x R} \rightarrow 0,
$$

induced by the natural projection. We have the long exact sequence

$$
\begin{aligned}
\operatorname{Tor}_{1}^{S}\left(S / I^{n+1} S, \mathfrak{m} / x \mathfrak{m}\right) & \rightarrow \operatorname{Tor}_{1}^{S}\left(S / I^{n+1} S, \mathfrak{n}\right) \rightarrow k \rightarrow \\
\frac{\mathfrak{m}}{I^{n+1} \mathfrak{m}+x \mathfrak{m}} & \rightarrow \frac{\mathfrak{m}}{I^{n+1} \mathfrak{m}+x R} \rightarrow 0 .
\end{aligned}
$$

Thus, the cokernel of the map $\operatorname{Tor}_{1}^{S}\left(S / I^{n+1} S, \mathfrak{m} / x \mathfrak{m}\right) \rightarrow \operatorname{Tor}_{1}^{S}\left(S / I^{n+1} S, \mathfrak{n}\right)$ has length at most one. If $\ell(I)>2$, then, by induction, the lengths of $\operatorname{Tor}_{1}^{S}\left(S / I^{n+1} S, \mathfrak{n}\right)$ are given by a polynomial of degree $\ell(I S)-1>0$, and since the length of $\operatorname{Tor}_{1}^{S}\left(S / I^{n+1} S, \mathfrak{m} / x \mathfrak{m}\right)$ is greater than or equal to $\lambda\left(\operatorname{Tor}_{1}^{S}\left(S / I^{n+1} S, \mathfrak{n}\right)\right)-1$, we are done. If $\ell(I)=2$, then by induction, if $\operatorname{Tor}_{1}^{S}\left(S / I^{n+1} S, \mathfrak{n}\right)=0$, then $I S$ is generated by a non-zerodivisor. Therefore $I$ is generated by a regular sequence of length two in $R$. But in this case, if we apply the Hilbert-Burch theorem to $I^{n}$, we readily see that $\operatorname{Tor}_{2}^{R}\left(R / I^{n}, k\right)=k^{n}$, so $\operatorname{deg}\left(\tau_{2}(n)\right)=1=\ell(I)-1$, which is what we want. Finally, note that when $\ell(I)=2$ and $\operatorname{Tor}_{1}^{S}\left(S / I^{n+1} S, \mathfrak{n}\right) \neq 0$, then the map $\operatorname{Tor}_{1}^{S}\left(S / I^{n+1} S, \mathfrak{n}\right) \rightarrow k$ cannot be surjective, for it would imply that the $\operatorname{map} \mathfrak{m} /\left(I^{n+1} \mathfrak{m}+x \mathfrak{m}\right) \rightarrow \mathfrak{m} /\left(I^{n+1} \mathfrak{m}+x R\right)$ is an isomorphism. This is false, as 
the non-zero class of the element $x$ gets mapped to zero. Thus, in this case, the lengths of $\operatorname{Tor}_{1}^{S}\left(S / I^{n+1} S, \mathfrak{m} / x \mathfrak{m}\right)$ are bounded below by a non-zero constant, and this finishes the proof of the proposition.

Remark 3.2. Before continuing, we would like to point out that for any finitely generated $R$-module $M$ and $I$-good filtration $\mathcal{J}$, if the lengths of $\operatorname{Tor}_{i}^{R}\left(R / J_{n}, M\right)$ are finite, then the function giving these lengths is a polynomial $\tau_{i}^{\mathcal{J}}(n)$ in $n$ for large $n$ and $\operatorname{deg}\left(\tau_{i}^{\mathcal{J}}(n)\right) \leq \ell(I)-1$. Indeed, let $K \subseteq F$ respectively denote the $i$ th syzygy and $(i-1)$ st free module in a resolution of $M$. Then $\operatorname{Tor}_{i}\left(R / J_{n}, M\right)=$ $\left(J_{n} F \cap K\right) / J_{n} K$. The graded module $\bigoplus_{n}\left(J_{n} F \cap K\right) / J_{n} K$ is a finite module over $\mathcal{R}_{I}$ which is annihilated by $\mathfrak{m}^{q} \mathcal{R}_{I}$ for some large, but fixed, value of $q$. Thus, the lengths in question are given by a polynomial of degree $\ell(I)-1$ or less. Note that if $\ell(I)=1$ and the lengths are not zero for $n$ large, then $\tau_{i}^{\mathcal{J}}(n)$ is a non-zero constant. In other words, in the case that $\ell(I)=1$, the polynomial $\tau_{i}^{\mathcal{J}}(n)$ has the "expected" degree $\ell(I)-1$.

As mentioned above, aside from the choice of filtration, our results depend upon the fact that the syzygies of $M$ have, among other properties, maximal dimension. Listed below are various conditions which guarantee the properties of syzygies that we need in Theorem 3.3 and Theorem 3.4.

\section{Conditions on $M, R$ and $I$.}

(a) $M$ has a rank (possibly zero).

(b) For all minimal primes $Q \subseteq R, M_{Q}$ is not a free $R_{Q}$-module.

(c) $R$ is equidimensional, unmixed and $\ell_{R / P}(I)=d$, for all minimal primes $P$.

(d) $M$ is a finite length $R$-module that tests finite projective dimension.

For $M$ as in condition (d), we mean that an $R$-module $N$ has projective dimension less than $i$ if and only if $\operatorname{Tor}_{i}^{R}(N, M)=0$. While the residue field $k$ obviously satisfies this condition (and this is the case we are mainly interested in), it follows from [1, Corollary 3.3], that $R / C$ satisfies the condition for any integrally closed m-primary ideal $C$.

Theorem 3.3. Let $M$ be a finitely generated $R$-module and $I \subseteq R$ be an ideal. Fix $i>0$. Let $\mathcal{J}:=\left\{J_{n}\right\}_{n \geq 0}$ be an I-good filtration of ideals such that the lengths of the modules $\operatorname{Tor}_{i}^{R}\left(R / J_{n}, M\right)$ are finite for $n$ large. Assume any one of the conditions (a), (b), (c) or (d). Then $\operatorname{deg}\left(\tau_{i}^{\mathcal{J}}(n)\right)=\ell(I)-1$ in each of the following cases:

(1) $J_{n}$ is divisible by $\mathfrak{m}$, i.e., there exists a good filtration $\left\{L_{n}\right\}$ with respect to $I$ such that $J_{n}=\mathfrak{m} L_{n}$ for all $n$.;

(2) $\left(\mathfrak{m} J_{n}: \mathfrak{m}\right)=J_{n}$ for all $n \gg 0$ and $\ell(I)=d$.

Proof. Let $K \subseteq F$ respectively denote the $i$ th syzygy and $(i-1)$ st free module in a minimal resolution of $M$, so that $\operatorname{Tor}_{i}\left(R / J_{n}, M\right)=\left(J_{n} F \cap K\right) / J_{n} K$. Now assume the filtration satisfies the condition in (1). Then since $K \subseteq \mathfrak{m} F, L_{n} K / \mathfrak{m} L_{n} K$ is contained in $\operatorname{Tor}_{i}^{R}\left(R / J_{n}, M\right)$, so in all cases, the degree of $\tau_{i}^{\mathcal{J}}(n)$ is greater than or equal to one less than the dimension of the graded module $\bigoplus_{n} L_{n} K / \mathfrak{m} L_{n} K$. By Proposition 2.2 above, this is $\ell_{R / \operatorname{ann}(K)}(I)$. If in all cases we show that $\ell(I)=\ell_{R / \operatorname{ann}(K)}(I)$, then $\operatorname{deg}\left(\tau_{i}^{\mathcal{J}}(n)\right) \geq \ell(I)-1$, which will give the result since $\operatorname{deg}\left(\tau_{i}^{\mathcal{J}}(n)\right) \leq \ell(I)-1$, by Remark 3.2. Now, if $M$ has a rank, then $K$ is faithful over $R$, so $\ell_{R / \text { ann }(K)}(I)=\ell(I)$. If for every minimal prime $Q \subseteq R, M_{Q}$ is not free, then $K_{Q} \neq 0$, so $\operatorname{ann}(K) \subseteq Q$. Thus $\operatorname{ann}(K)$ is nilpotent. It follows easily from this that $\ell_{R / \operatorname{ann}(K)}(I)=\ell(I)$. If 
$R$ is equidimensional and unmixed, then since any prime minimal over the annihilator of $K$ must be in $\operatorname{Ass}(R)$, it follows that $\operatorname{dim}(R / \operatorname{ann}(K))=d=\operatorname{dim}(R)$. Since $I$ extended to $R / \operatorname{ann}(K)$ still has maximal analytic spread modulo each minimal prime, we have $\ell_{R / \operatorname{ann}(K)}(I)=d=\ell(I)$. Finally, suppose $M$ satisfies condition (d). If $\operatorname{depth}(R)>0$, then $\operatorname{rank}(M)=0$, and this case is covered by case (a). Suppose $\operatorname{depth}(R)=0$. Then, on the one hand, we have that $K_{Q}$ is a free summand of $F_{Q}$ of constant rank for all prime ideals $Q \neq \mathfrak{m}$. If $K_{Q}=0$ for all $Q$, then $K$ has finite length. Thus $J_{p} \cdot K=0$, for $p \gg 0$. It follows that $\operatorname{Tor}_{i}^{R}\left(R / J_{n}, M\right)=0$, for $n$ large. On the other hand, by condition (d), the vanishing of Tor against $M$ implies that $R / J_{n}$ has finite projective dimension, which is absurd, $\operatorname{since} \operatorname{depth}(R)=0$. Thus, $K_{Q} \neq 0$ for all minimal primes $Q$, so $\operatorname{ann}(K)$ is nilpotent. Therefore, $\ell_{R / \operatorname{ann}(K)}(I)=\ell(I)$. Thus, in all cases, $\ell_{R / a n n(K)}(I)=\ell(I)$, so in all cases $\operatorname{deg}\left(\tau_{i}^{\mathcal{J}}(n)\right)=\ell(I)-1$.

Now assume the filtration satisfies the condition (2). Because $\bigoplus_{n}\left(J_{n} F \cap K\right)$ is a finite module over $\mathcal{R}_{I}$, there exists $t \geq 1$ such that $J_{n} F \cap K=I^{n-t}\left(J_{t} F \cap K\right)$, for $n \geq t$. It follows that $I^{t}$ annihilates $\operatorname{Tor}_{i}^{R}\left(R / J_{n}, M\right)$ for all $n \geq t$. Increasing $t$ if necessary, we may replace $I$ by $I^{t}$ and the filtration $\mathcal{J}$ by $\left\{J_{n t}\right\}$, and after changing notation, assume that for all $n \geq 1, J_{n} F \cap K=I\left(J_{n-1} F \cap K\right)$, I annihilates $\operatorname{Tor}_{i}^{R}\left(R / J_{n}, M\right)$ and $\left(\mathfrak{m} J_{n}: \mathfrak{m}\right)=J_{n}$.

We now have $\operatorname{Tor}_{i}^{R}\left(R / J_{n}, M\right)=I\left(J_{n-1} F \cap K\right) / J_{n} K \subseteq J_{n-1} K / J_{n} K$, since $I$ annihilates $\operatorname{Tor}_{i}^{R}\left(R / J_{n-1}, M\right)$. It follows that the socle of $\operatorname{Tor}_{i}^{R}\left(R / J_{n}, M\right)$ is contained in the socle of $J_{n-1} K / J_{n} K$. On the other hand, let $v \in J_{n-1} K$ be such that its image in $J_{n-1} K / J_{n} K$ is contained in the socle. Then $\mathfrak{m} \cdot v \in J_{n} K$. Thus, $\mathfrak{m} \cdot v \in \mathfrak{m} J_{n} F$. Our hypothesis on $J_{n}$ implies that $v \in J_{n} F$. Thus $v \in J_{n} F \cap K$, so the image of $v$ in $J_{n-1} K / J_{n} K$ is in the socle of $\operatorname{Tor}_{i}^{R}\left(R / J_{n}, M\right)$. In other words, the socle of $\operatorname{Tor}_{i}^{R}\left(R / J_{n}, M\right)$ equals the socle of $J_{n-1} K / J_{n} K$. Therefore, the degree of $\tau_{i}^{\mathcal{J}}(n)$ is greater than or equal to the degree of the polynomial which gives the lengths of the socles of $J_{n-1} K / J_{n} K$. To finish, we must calculate the dimension of $\bigoplus_{n \geq 0} \operatorname{Soc}\left(J_{n} K / J_{n+1} K\right)$ as a module over $\mathcal{R}_{I}$. This calculation is similar in spirit to the one given for $\mathrm{H}_{\mathfrak{m}}^{0}(-)$ of a graded module in the comment after Definition 6.1.1 in [2].

On the one hand, our hypotheses on $M$ imply that $\operatorname{dim}(K)=d$, so by Proposition 2.2 , we have $\operatorname{dim}\left(\bigoplus_{n \geq 0} J_{n} K / J_{n+1} K\right)=d$. On the other hand, the hypotheses on $I$ and $M$ yield $\ell_{K}(I)=d$, so by Proposition 2.2, $\bigoplus_{n \geq 0} J_{n} K / \mathfrak{m} J_{n} K$ has dimension $d$. Take $Q$ to be a prime ideal in $\mathcal{R}_{I}$ of dimension $d$ that is minimal over the annihilator of $\bigoplus_{n \geq 0} J_{n} K / \mathfrak{m} J_{n} K$. We claim that $Q$ contains the annihilator of $\bigoplus_{n \geq 0} J_{n} K / J_{n+1} K$. Suppose not. Let $f \in \mathcal{R}_{I}$ have degree $r$ and suppose $f \notin$ $Q$, but $f$ is in the annihilator of $\bigoplus_{n \geq 0} J_{n} K / J_{n+1} K$. Note that $r \neq 0$, since $Q$ contains $\mathfrak{m}$. Increasing $r$ if necessary, we may assume $J_{n+1+r} K=I J_{n+r} K$, for all $n$. Thus, for all $n, f \cdot J_{n} K \subseteq J_{n+1+r} K \subseteq \mathfrak{m} J_{n+r} K$, so $f$ belongs to the annihilator of $\bigoplus_{n \geq 0} J_{n} K / \mathfrak{m} J_{n} K$, a contradiction. Thus, the claim holds, so $Q \in$ $\operatorname{Ass}\left(\bigoplus_{n \geq 0} J_{n} K / J_{n+1}^{-\infty} K\right)$.

If we write $Q=(0: f)$, for $f \in \bigoplus_{n \geq 0} J_{n} K / J_{n+1} K$, it follows that $f$ belongs to the graded module $\bigoplus_{n \geq 0} \operatorname{Soc}\left(J_{n} K / J_{n+1} K\right)$. Thus, the dimension of this module is $d$, so the polynomial giving the lengths of the modules $\operatorname{Soc}\left(J_{n} K / J_{n+1} K\right)$ has degree $d-1$. Thus, $\operatorname{deg}\left(\tau_{i}^{\mathcal{J}}(n)\right) \geq d-1$. Since the reverse inequality always holds, the proof of part (2) is complete. 
Theorem 3.4. Let $M$ be a finitely generated $R$-module and $I \subseteq R$ an ideal. Fix $i>0$. Let $\mathcal{J}:=\left\{J_{n}\right\}_{n \geq 0}$ be an I-good filtration of ideals such that the lengths of the modules $\operatorname{Tor}_{i}^{R}\left(R / J_{n}, M\right)$ are finite for $n$ large. Assume $R$ is quasi-unmixed and that any one of the module conditions (a), (b) or (d) holds. In addition, suppose that $\mathcal{R}_{\mathcal{J}}$ is a ring and $\left(J_{n}: \mathfrak{m}\right) \nsubseteq \overline{J_{n}}$ for some $n$. Then $\operatorname{deg}\left(\tau_{i}^{\mathcal{J}}(n)\right)=\ell(I)-1$.

Proof. Let $\mathcal{L}$ denote the filtration $\left\{L_{n}\right\}$ whose $n$th term is $L_{n}:=\left(J_{n}: \mathfrak{m}\right)$. We first note that the assumption that $L_{n} \not \subset \overline{J_{n}}$ for some $n$ implies that $\ell(I)=d$, so that our goal is to show that $\operatorname{deg}\left(\tau_{i}^{\mathcal{J}}(n)\right)=d-1$. Indeed, if say $L_{q} \not \subset \overline{J_{q}}, \mathfrak{m}$ is an associated prime of $R / \overline{J_{q}}=R / \overline{I^{q}}$, and hence $\mathfrak{m}$ is associated to $R / \overline{I^{n}}$ for all $n \geq q$ (7], Proposition 3.4), so $\ell(I)=d$ ( [7, Proposition 4.1).

As before, let $K \subseteq F$ respectively denote the $i$ th syzygy and $(i-1)$ st free module in a minimal resolution of $M$, so that $\operatorname{Tor}_{i}\left(R / J_{n}, M\right)=\left(J_{n} F \cap K\right) / J_{n} K$. We now claim that the graded module $\bigoplus_{n>0} L_{n} K / J_{n} K$ has dimension $d$. Suppose the claim holds. Then the degree of the polynomial giving the lengths of the components of this module has degree $d-1$. Since $K \subseteq \mathfrak{m} F$, we have $L_{n} K / J_{n} K \subseteq$ $\operatorname{Tor}_{i}^{R}\left(R / J_{n}, M\right)$, so $\operatorname{deg}\left(\tau_{i}^{\mathcal{J}}(n)\right) \geq d-1$. Since the reverse inequality always holds, we have $\operatorname{deg}\left(\tau_{i}^{\mathcal{J}}(n)\right)=d-1$.

To verify the claim, we first note that the proof of Theorem 3.3 shows that in each of the cases (a), (b) and (d), the annihilator of $K$ is nilpotent. Suppose first that $\operatorname{depth}(R)>0$. Note that this automatically holds in case (a). Then there is a non-zerodivisor $x \in \mathfrak{m}$. It follows that $x \cdot \mathcal{R}_{\mathcal{L}} \subseteq \mathcal{R}_{\mathcal{J}}$, so the filtrations $\mathcal{J}$ and $\mathcal{L}$ satisfy the requirements of Proposition 2.3. Thus, by Proposition 2.3, the dimension of the graded module $\bigoplus_{n \geq 0} L_{n} K / J_{n} K$ is equal to $d$.

Suppose that $\operatorname{depth}(R)=0$. We reduce to the case of positive depth as follows. Let $U$ denote the $\mathfrak{m}$-torsion part of $R$, i.e., the set of elements in $R$ annihilated by some power of $\mathfrak{m}$. Then $U$ is a nilpotent ideal and $S:=R / U$ is a quasi-unmixed local ring with positive depth. Writing $I S, \mathcal{J} S$ and $\mathcal{L} S$ for extensions to $S$, we

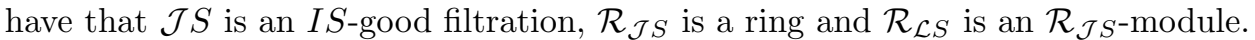
Moreover, since $\bigoplus_{n>0} L_{n} S \subseteq \bigoplus_{n>0}\left(J_{n} S:_{S} \mathfrak{m} S\right)$, the filtration $\mathcal{L} S$ meets the third requirement of Proposition 2.3; i.e., there exists a non-zerodivisor $x \in S$ such that $x \cdot \mathcal{R}_{\mathcal{L} S} \subseteq \mathcal{R}_{\mathcal{J} S}$. If we choose $q$ so that $L_{q} \nsubseteq \overline{J_{q}}$, we also have that $L_{q} S \nsubseteq \overline{J_{q} S}$. Since the annihilator of the $S$-module $K / U K$ is readily seen to be nilpotent, Proposition 2.3 yields that the $\mathcal{R}_{I S \text {-module }} \bigoplus_{n \geq 0}\left(L_{n} K+U K\right) /\left(J_{n} K+U K\right)$ has dimension $d$. Thus, $\bigoplus_{n \geq 0} L_{n} K / J_{n} K$ has dimension $d$, which is what we want.

The following corollary follows from the first part of Theorem 3.3 and shows that Kodiyalam's question has a positive answer when we consider the Betti numbers of $\mathfrak{m} I^{n}$ or the Betti numbers of $I^{n}$ for $I$ divisible by $\mathfrak{m}$.

Corollary 3.5. Let $I \subseteq R$ be an ideal, $i>0$ and $M$ a finite $R$-module. Assume that any one of the module conditions (a), (b), (c) or (d) is in place. Then $\tau_{i}^{\mathcal{J}}(n)$ has degree $\ell(I)-1$ in each of the following cases : (i) $\mathcal{J}:=\left\{\mathfrak{m} I^{n}\right\}$ for all $n$ and (ii) $\mathcal{J}:=\left\{I^{n}\right\}$, with $I=\mathfrak{m} L$ for some ideal $L$, provided the modules $\operatorname{Tor}_{i}^{R}\left(R / J_{n}, M\right)$ have finite length. In particular, for any ideal $I$ and $i>0$, the ith Betti numbers of $R / \mathfrak{m} I^{n}$ and $\mathfrak{m} I^{n}$ are given by polynomials having degree $\ell(I)-1$ and if $I=\mathfrak{m} L$, for some ideal $L$, then the Betti numbers of $R / I^{n}$ and $I^{n}$ are given by polynomials having degree $\ell(I)-1$. 
Proof. The first statement is clear. For the second statement, set $L_{n}:=\mathfrak{m}^{n-1} L^{n}$, so that $\left\{L_{n}\right\}$ is an $I$-good filtration; taking $J_{n}=I^{n}=\mathfrak{m} L_{n}$, Theorem 3.3(1) applies.

Note that if $I=\mathfrak{m}$, then it follows from Corollary 3.5 (or Theorem 3.3) that if $R$ is unmixed and equidimensional, then $\tau_{i}^{\mathfrak{m}}(n)$ has degree $d-1$. This greatly extends [3, Theorem I]. Moreover, it follows that the polynomial giving the Betti numbers of $\mathfrak{m}^{n}$ has degree $d-1$, so we have a simpler proof of Kodiyalam's result ([4, Example 12) when $N=R$.

It follows from our next corollary that Kodiyalam's question has a positive answer for the filtration $\left\{\overline{I^{n}}\right\}$ when $R$ is analytically unramified and $I$ has maximal analytic spread.

Corollary 3.6. For $I \subseteq R$ and $M$ a finite $R$-module as in the previous corollary, assume $\ell(I)=d$. Then $\operatorname{deg}\left(\tau_{i}^{\mathcal{J}}(n)\right)=d-1$ in the following cases:

(1) $\mathcal{J}=\left\{\left(\mathfrak{m} I^{n}: \mathfrak{m}\right)\right\}$ and $\operatorname{depth}(R)>0$,

(2) $\mathcal{J}=\left\{\left(I^{n}: \mathfrak{m}\right)\right\}$ and $\operatorname{depth}(R)>0$,

(3) $R$ is analytically unramified and $\mathcal{J}=\left\{\overline{I^{n}}\right\}$,

provided the modules $\operatorname{Tor}_{i}^{R}\left(R / J_{n}, M\right)$ have finite length. In particular, for $n$ large, the ith Betti numbers of $\left(\mathfrak{m} I^{n}: \mathfrak{m}\right),\left(I^{n}: \mathfrak{m}\right)$ and $\overline{I^{n}}$ (when $R$ is analytically unramified) are given by polynomials of maximal degree.

Proof. In each case $\mathcal{R}_{\mathcal{J}}$ is finite over $R_{I}$, so the filtration is good with respect to I. Moreover, in each of these cases, the filtration satisfies $\left(\mathfrak{m} J_{n}: \mathfrak{m}\right)=J_{n}$ for all $n$, so the second part of Theorem 3.3 applies.

Remark 3.7. Let $R$ be an analytically unramified local ring and $I \subseteq R$ an ideal. It is well known that there exists $t \geq 1$ such that $J:=\overline{I^{t}}$ is a normal ideal, i.e., the powers of $J$ are all integrally closed. Thus if $\ell(I)=d$, then $\operatorname{deg}\left(\tau_{i}^{\mathcal{J}}(n)\right)=d-1$, for $\mathcal{J}$ the $J$-adic filtration. In particular, the $i$ th Betti numbers of the powers of $J$ are given by a polynomial of maximal degree. In fact, a stronger result follows from Corollary 3.8.

The following corollary is an immediate consequence of Theorem 3.4.

Corollary 3.8. Let $R$ be a quasi-unmixed local, $I \subseteq R$ be an ideal, and let $M$ be a finitely generated $R$-module. Assume any one of the module conditions (a), (b) or (d). If $\left(I^{n}: \mathfrak{m}\right) \nsubseteq \overline{I^{n}}$ for some $n$, then for all $i>0$, if the modules $\operatorname{Tor}_{i}\left(R / I^{n}, M\right)$ have finite length, $\tau_{i}^{I}(n)$ has maximal degree $d-1$. It follows that the ith Betti numbers of $R / I^{n}$ and $I^{n}$ are given by polynomials having degree $d-1$. In particular, if $I$ is an integrally closed ideal and $\mathfrak{m} \in A s s(R / I)$, then $\tau_{i}^{I}(n)$ has maximal degree and the ith Betti numbers of $R / I^{n}$ and $I^{n}$ are given by polynomials having degree $d-1$, for all $i>0$.

Remark 3.9. Two comments regarding the previous corollary are in order. The first is that, as in the proof of Theorem 3.4, the condition $\left(I^{n}: \mathfrak{m}\right) \nsubseteq \overline{I^{n}}$ for some $n$ implies that $I$ has analytic spread $d$. The second comment is that, for $I \subseteq R$ an $\mathfrak{m}$-primary ideal, the condition $\left(I^{n}: \mathfrak{m}\right) \nsubseteq \overline{I^{n}}$ for some $n$ (and hence all large $n$ ) seems to hold more often than not, in a sense that we have not been able to quantify. Indeed, if this condition doesn't hold, then $\left(I^{n}: \mathfrak{m}\right) \subseteq \overline{I^{n}}$ for all $n$. The only examples we know of satisfying this latter condition are ideals $I$ which also 
have the property that $I^{n}$ has finite projective dimension for $n$ large. Of course, then $\tau_{i}^{I}(n)$ is identically zero when $i>d$.

Note added in proof. S. Goto has shown us an example of an ideal $I$ generated by a system of parameters in a Buchsbaum local ring $R$ so that $\left(I^{n}: \mathfrak{m}\right) \subseteq \overline{I^{n}}$ for all $n$ and $I^{n}$ has infinite projective dimension for all $n$.)

It should be clear from the foregoing, that in those (rare) cases where syzygies of $M$ over $R$ have small dimension, one cannot expect $\operatorname{deg}\left(\tau_{i}(n)\right)$ to equal $\ell(I)-1$. This phenomenon is at the heart of the example given in [3]. In fact, let $R$ be any local ring with finite module $M$ that has an $i$ th syzygy $K$ of dimension less than $d$. Then, say for any $\mathfrak{m}$-primary ideal $I$, after replacing $I$ by large powers of itself, $\operatorname{Tor}_{i}^{R}\left(R / I^{n}, M\right) \subseteq I^{n-1} K / I^{n} K$ (as in the proof of Theorem 3.3), and it follows that $\operatorname{deg}\left(\tau_{i}(n)\right) \leq \operatorname{deg}\left(P_{K}(n)\right)<d-1$, where $P_{K}(n)$ is the Hilbert polynomial giving the lengths of $I^{n-1} K / I^{n} K$. This can be realized concretely (as in [3]) by finding a ring $R$ containing an ideal $J$ such that $\operatorname{dim}(J)<d$ and taking $M=R / J$ and $i=1$. On the other hand, as pointed out in Remark 1.1, for any local ring $R$, every syzygy of $k$ has nilpotent annihilator, so one cannot produce a similar example with $K$ a syzygy of $k$. This lends further credence for a positive answer to Kodiyalam's question.

\section{REFERENCES}

1. A. Corso, C. Huneke, D. Katz and W. Vasconcelos, Integral closure of ideals and annihilators of homology, Lecture Notes in Pure and Applied Mathematics 227 (2005), 33-48. MR.2184788 (2006h:13015)

2. H. Flenner, L. O'Carroll, and W. Vogel, Joins and Intersections, Springer Monographs in Mathematics, Springer Verlag, Berlin, 1999. MR1724388 (2001b:14010)

3. S. Iyengar and T. Purthenpurakal, Hilbert-Samuel functions of modules over Cohen-Macaulay rings, Proc. Amer. Math. Soc. 135 (2007), 637-648.

4. V. Kodiyalam, Homological invariants of powers of an ideal, Proc. Amer. Math. Soc. 118, No. 3 (1993), 757-764. MR 1156471 (93i:13022)

5. G. Levin, Local rings and Golod homomorphisms, J. Algebra 37 (1975), 266-289. MR0429868 $(55: 2878)$

6. T. Marley, Hilbert Functions in Cohen-Macaulay Rings, Ph.D. Dissertation, Purdue University, 1989.

7. S. McAdam, Asymptotic Prime Divisors, Lecture Notes in Math., vol. 1023, Springer-Verlag, New York, 1983. MR.0722609 (85f:13018)

8. L.J. Ratliff, Jr., On quasi-unmixed semi-local rings and the altitude formula, Amer. J. Math. 87 (1965), 278-284. MR0179199 (31:3448)

9. L.J. Ratliff, Jr., Locally quasi-unmixed Noetherian rings and ideals of the principal class, Pac. J. Math. 52 (1974), 185-205. MR0352085 (50:4573)

10. E. Theodorescu, Derived functors and Hilbert polynomials, Math. Proc. Camb. Phil. Soc. 132 (2002), 75-88. MR.1866325 (2002j:13018)

Department of Mathematics, University of Kansas, Lawrence, Kansas 66045

E-mail address: dlk@math.ku.edu

Department of Mathematics, University of Missouri, Columbia, Missouri, 65211

E-mail address: theodore@math.missouri.edu

Current address: University of Iowa, Department of Actuarial Science and Statistics, 241

Schaeffer Hall, Iowa City, IA 52242

E-mail address: emanoil.theodorescu@g.mail.com 